\title{
LAS RELACIONES ENTRE LAS EMPRESAS COTIZADAS ESPANOLAS: UN ANALISIS A TRAVES DE LAS CONSEJERIAS CRUZADAS
}

\author{
CARLOS SICILIA \\ JOSE M. SALLAN \\ DEPARTAMENTO DE ORGANIZACION DE EMPRESAS \\ ESCUELA TECNICA SUPERIOR DE INGENIERIAS INDUSTRIALY AERONAUTICA DETERRASSA \\ UNIVERSIDAD POLITECNICA DE CATALUNYA
}

\begin{abstract}
Resumen: El objetivo de este trabajo es analizar las características de la organización económica de la empresa española, mediante el análisis sociométrico de la red de consejerías cruzadas de las empresas españolas más importantes a finales del año 2005, cotizadas en el IBEX 35. Con este fin, se ha obtenido la red social definida por las consejerías cruzadas en las empresas cotizadas, y se ha analizado mediante métricas de centralidad, así como de obtención de agrupamientos. Dichos agrupamientos se han obtenido mediante el procedimiento CONCOR. Los resultados del análisis indican que la red de empresas españolas define un modelo peculiar de organización económica, en el que el papel central no corresponde a los bancos, sino a las empresas públicas privatizadas en los años noventa. Comparando estos resultados con los de estudios anteriores, podemos concluir que el gobierno de las empresas españolas está en un momento de transición. Su evolución futura dependerá de la evolución de las compañías privatizadas, y de la posición de las entidades bancarias respecto del negocio de banca industrial.
\end{abstract}

Palabras clave: Gobierno corporativo, consejerías cruzadas, redes sociales.

\section{Introducción y objetivos}

Una cuestión importante para el progreso económico de cualquier país es que sus empresas estén bien gobernadas. La teoría del gobierno de la em- presa trata de definir mecanismos por los cuales quienes proporcionan a la empresa fondos financieros se aseguren un retorno adecuado a sus inversiones (Shleifer y Vishny, 1997). Uno de los mecanismos para asegurar el buen gobierno de la empresa es el consejo de administración, en el que la junta de accionistas delega la dirección de la empresa. Una de las funciones más relevantes del consejo de administración, especialmente en sociedades cotizadas en las que existe una clara separación entre dirección y control, es la función de control: en este tipo de sociedades, el consejo de administración actúa como un instrumento para minimizar los costes de agencia de los accionistas (Fama y Jensen, 1983). Pero además de la función de control, los consejos de administración realizan otras dos funciones: la función estratégica y la función de servicio (Zahra y Pierce, 1989). La función estratégica consiste en asesorar a la dirección en la elaboración de la estratégica, y en participar en la aprobación final de ésta. Los miembros del consejo realizan la función de servicio cuando permiten a la empresa acceder a recursos difíciles de obtener de otro modo, por ejemplo financiación o información. En el modelo de gobierno corporativo español, estas tres funciones son realizadas por un único órgano de gobierno. En este sentido, el modelo español es similar al anglosajón. En otros modelos de gobierno, como el alemán, existen dos órganos de gobierno: consejo de vigilancia o Aufsichtsrat, dedicado a la función de control, y el con- 
sejo directivo o Vorstand, que desarrolla el resto de funciones (Prowse, 1994). En España, las cajas de ahorros tienen un sistema de gobierno similar al alemán (Azofra y Santamaría. 2004).

Una de las formas en que los consejeros de administración pueden llevar a cabo su función de servicio es participando en más de un consejo de administración. Los consejeros que participan en el gobierno de varias empresas conforman una red social, que es reflejo y a la vez determina parcialmente la organización económica de las empresas más significativas de un país. El objetivo de este trabajo es analizar las características de la organización económica española, mediante el análisis sociométrico de la red de consejerías cruzadas de las grandes empresas cotizadas españolas.

\section{Concepto de consejería cruzada y sus consecuencias}

Se produce una cruce de consejerías (interlocking directorate) cuando un miembro del consejo de administración de una empresa ocupa un puesto en el consejo en otra empresa distinta. Cabe preguntarse sobre las causas que llevan al establecimiento de los cruzamientos. Tales causas han sido descritas en la literatura (Zajac, 1988; Mizruchi, 1996; Haunschild y Beckman, 1998) y algunas corroboradas empíricamente con más o menos éxito (ver, por ejemplo, O'Hagan, 2004). También podemos preguntarnos por las consecuencias que puedan acarrear: en algunos países las consejerías cruzadas están limitadas por la legislación, en función de los actores entre los que se establecen.

Una posible justificación de la existencia de consejerías cruzadas podría ser la reducción de competencia. Al compartir consejeros, dos o más empresas de un mismo sector podrían ponerse de acuerdo con más facilidad para tomar medidas que redujeran la competencia. Sin embargo, no hay evidencia de que las empresas con mayor número de consejeros cruzados con otras empresas del mismo sector consigan mayores cuotas de mercado o mayores beneficios (Pennings, 1980). El término cooptación tiene un sentido doble, el que se utiliza habitualmente para hablar de los consejos de administración se refiere al reclutamiento de consejeros a partir del voto favorable de los otros consejeros. En la literatura sobre consejerías cruzadas se le asigna un significado más amplio, asociado al ingreso de fuentes de incertidumbre al dominio de la empresa. Determinados consejeros se incorporan al consejo de una deter- minada empresa por ser consejeros de otras entidades que controlan algún recurso vital para ésta, principalmente el capital. Así una empresa puede proponer que un consejero de una entidad bancaria con la que tiene una deuda se incorpore al consejo (Mintz y Schwartz, I981; Byrd y Mizruchi, 2005). De esta forma ambas empresas refuerzan los lazos aumentando la seguridad de la inversora y de la invertida. Para la entidad inversora el consejero enviado ejercerá una clara función de control, mientras que la entidad receptora se asegura, hasta cierto punto, el acceso al capital necesario gracias a la presencia del consejero cruzado. Según la teoría del control bancario, si el recurso a cooptar consejeros de entidades bancarias se generaliza entre las empresas de un sistema, las entidades bancarias ( $y$ en menor medida, las aseguradoras) tendrán una posición central en la red social de consejerías cruzadas (Mintz y Schwartz, | 981), y por tanto una gran influencia en la organización económica del país. Además de financiación, los consejeros cruzados pueden aportar otros recursos. Por ejemplo, pueden actuar como puentes tendidos entre las empresas por los que fluye el conocimiento sobre las mejores prácticas, que serían imitadas rápidamente por las más cercanas en la red de consejeros cruzados (Haunschild, 1993). Si la red de consejerías es lo suficientemente densa, el resultado podría ser una organización económica altamente institucionalizada, en la que las empresas actuarían de forma muy similar (DiMaggio y Powell, 1983). Finalmente, los consejeros cruzados pueden aportar prestigio. El consejero de una empresa que pertenece a otra mayor, de más prestigio o de mejor desempeño proporciona a la primera una imagen de solidez y de buena gestión ante los stakeholders. Por esta razón, las empresas pueden intentar incorporan consejeros de reconocido prestigio en el campo de la gestión y no necesariamente en el mismo sector.

Del mismo modo que un consejero cruzado pone en contacto a dos empresas, también podemos considerar que dos consejeros entran en contacto desde el momento en que se sientan en el mismo consejo. Tendríamos entonces una red de consejeros (Zajac, 1988), en la que los consejeros cruzados tendrían, con toda probabilidad, una alta centralidad. Estos consejeros podrían tener una gran influencia en la red social de empresas. Los consejeros que pertenecen a varios consejos son mejor valorados, no solamente por la imagen que ofrecen sino también por la experiencia que adquieren. $Y$ cuanto mayor sea el currículum de pertenencias a consejos, tanto mayor será la probabilidad de que el consejero sea invitado a participar en otros nuevos (Davis, 1993). 
Además, el círculo social en el que se mueven los consejeros de las empresas de características similares les hace compartir otros ambientes que no son las salas de reuniones. El círculo se estrecha en momentos compartidos en la vida privada de los consejeros: los clubes de golf, por ejemplo, se convierten en lugares donde afianzar estos lazos. Según la teoría del control directivo (Useem, 1984), los mecanismos que hemos descrito harían que un pequeño núcleo de consejeros tuviera una gran influencia sobre la organización económica de un país.

En un estudio previo sobre esta misma cuestión, Aguilera (1998) evalúa la red social definida por las consejerías cruzadas de las empresas españolas más importantes en 1993. Según esta autora, en ese momento el gobierno de las empresas españolas respondía al modelo continental, en el que los bancos y las compañías de servicios tenían una elevada centralidad en la red, y en la cual los vínculos entre empresas son más fuertes entre empresas de sectores distintos que dentro de un mismo sector. Con este trabajo, reevaluaremos la red social en 2005, teniendo así una visión longitudinal de la evolución del sistema de grandes empresas españolas.

\section{Muestra y datos}

Para este estudio hemos recopilado información sobre los consejeros de las empresas que formaban parte del selectivo IBEX 35 al terminar el año 2005. El índice IBEX 35 se compone de los 35 valores cotizados con mayor liquidez en el periodo. Las empresas del IBEX 35 son, pues, sociedades cotizadas seleccionadas por un Comité AsesorTécnico que valora otros criterios como la dimensión de las empresas candidatas y la calidad de las transacciones realizadas. La muestra sin embargo no se limita a las 35 empresas del IBEX: hemos decidido incluir en el estudio dos actores que a lo largo de la recogida de datos se han revelado como potencialmente significativos. A pesar de no ser sociedades cotizadas, las cajas de ahorros de España cuentan con una estructura de gobierno corporativo que las hace susceptibles de ser analizadas conjuntamente con las que sí lo son. Como ya hemos avanzado más arriba, cuentan con un doble sistema de consejos, uno para las tareas de control y otro para las tareas estratégicas. Hemos decidido su inclusión en la red por su ubicuidad en el círculo de consejerías cruzadas de las empresas del IBEX.

Para obtener los datos de consejeros cruzados se ha recurrido a dos fuentes de información. En primer lugar se ha acudido a las propias empresas objeto de estudio. Dado que el código Olivencia sugiere la necesidad de transparencia en la gestión de las empresas cotizadas, la mayoría de las empresas con cierta preocupación por las prácticas de buen gobierno se preocupan de publicar la información relativa a sus órganos de dirección. Para ello, aprovechando la publicidad de tal información hemos acudido a las páginas web corporativas de cada una de ellas donde, en la mayoría de casos bajo el epígrafe de «relaciones con los accionistas», se da cuenta de la composición del consejo de administración.

En algunos casos, cuando se ha buscado la información a posteriori y en la web no se encontraba el histórico, o bien cuando no se ha encontrado la información sobre el consejo en la propia web de la empresa, se ha recurrido a la Comisión Nacional del Mercado de Valores. Efectivamente, las empresas remiten regularmente (una vez al año) un informe sobre gobierno corporativo a esta Comisión, que también se puede consultar públicamente en su página web. En tal informe, redactado según un modelo similar para todas las empresas, se recoge información diversa sobre el consejo, los consejeros, las prácticas de gobierno corporativo y los hechos significativos que han acaecido durante el periodo al que el informe se refiere. Se entiende por hecho significativo, por ejemplo el cambio de consejeros o del papel que estos desempeñan en el consejo.

\section{Análisis y resultados}

Los datos se recogen en una matriz rectangular (Scott, 2000), de $m$ filas y n columnas, siendo $m$ el número de consejeros y $n$ el de empresas que forman parte de la muestra. El elemento $(i, j)$ de la matriz valdrá uno si el consejero i forma parte del consejo de la empresa j, y cero en caso contrario. En la segunda fase del análisis pueden construirse dos matrices simétricas de adyacencia, una para los consejeros y otra para las empresas. Para el análisis hemos empleado la segunda matriz. Es una matriz cuadrada de orden $n$, en la que su elemento $(i, j)$ es igual al número de consejeros que comparten las empresas $i$ y j. Si obviamos los elementos de la diagonal, a partir de esta matriz obtenemos un grafo no orientado, en el que los nodos representan las empresas, y las aristas las conexiones entre empresas a través de consejeros cruzados. Cada arista del grafo tendrá un peso igual al número de consejeros que comparten las empresas unidas por dicha arista. A partir de esta matriz de empresas Ilevaremos a cabo dos análi- 
Tabla I

Entidades más centrales en la red de empresas según el grado en el grafo ponderado y una vez dicotomizada (primer cuartil)

\begin{tabular}{|l|c|c|c|}
\hline \multicolumn{1}{|c|}{ Entidad } & Grado (grafo ponderado) \\
dicotornizado) & Entidad & Grado (grafo \\
\hline Telefónica & 16 & Iberia & 10 \\
Iberia & 12 & Repsol & 9 \\
Repsol & Enagás & 9 \\
Enagás & Telefónica & 8 \\
Sogecable & Unión Fenosa & 7 \\
Telefónica Móviles & 10 & Abertis & 7 \\
ACS & 10 & ACS & 7 \\
Abertis & 10 & Endesa & 6 \\
Prisa & 10 & Santander CE & 6 \\
\end{tabular}

sis sociométricos: la detección de los actores centrales en el sistema mediante las medidas más usuales de centralidad, y el estudio de la estructura de la red, buscando agrupaciones de empresas con un perfil de conexiones similar.

\section{I. Centralidad}

Algunas de las teorías sobre consejerías cruzadas, como la del control bancario, se basan en la naturaleza de los individuos centrales en la red. Por tanto, resulta pertinente evaluar a los actores mediante algunas métricas de centralidad. A pesar de que la centralidad en una red social se puede medir de múltiples formas (Freeman, 1979; Ashar y Shapiro, 1988; Freeman, Borgatti y White, 1991; Marsden, 2002), para este análisis utilizaremos dos variantes de la centralidad de grado (Freeman, 1979). La primera variante es el grado del nodo en el grafo ponderado. Será igual, por tanto, al número de consejerías cruzadas de la empresa con otras empresas (un mismo consejero puede dar lugar a más de una consejería cruzada, si está presente en tres o más consejos de empresas de la muestra). Como se ha observado que algunas empresas comparten un número elevado de consejeros (algunos consejos de administración de la muestra comparten cinco o más consejeros), hemos considerado también un grafo dicotomizado, en el que los pesos de todas las aristas son iguales a uno. La centralidad de grado para un nodo de este grafo será igual, por tanto, al número de empresas con las que comparte consejeros la empresa asociada al nodo considerado. De este modo, podremos saber cuáles son las empresas que más relaciones establecen con otras, independientemente del número de consejeros que compartan. Podemos obtener una perspectiva diferen- te de centralidad computando la centralidad de proximidad o closeness centrality (Freeman, 1979). La centralidad de grado representa un índice de centralidad local, en el sentido de que viene determinada exclusivamente por la posición de los actores frente a los que le son más cercanos, aquéllos con los que cruza consejeros directamente. La centralidad de proximidad se computa a partir de los posibles caminos que unen los diferentes actores. Esta segunda forma de medir la centralidad permite evaluar la centralidad de los actores de la red de una forma más global.

Para el cálculo de las medidas de centralidad se ha utilizado el programa UCINET (Borgatti, Everett y Freeman, 2002). En la Tabla I se muestran los resultados del cálculo de centralidad de grado para el grafo ponderado y para el dicotomizado.

En la Tabla 2 tenemos los valores de centralidad de proximidad para el primer cuartil de la muestra. El valor numérico corresponde al valor normalizado obtenido con el software UCINET.

Tabla 2

Entidades más centrales en la red de empresas según proximidad

\begin{tabular}{|l|c|}
\hline \multicolumn{1}{|c|}{ Entidad } & Proximidad (normalizado) \\
\hline Iberia & 14,94 \\
Repsol & 14,69 \\
Enagás & 14,63 \\
Teléfonica & 14,63 \\
Unión Fenosa & 14,63 \\
Abertis & 14,52 \\
Teléfonica Móviles & 14,46 \\
ACS & 14,40 \\
NHHoteles & 14,34 \\
\hline
\end{tabular}


Tabla 3

Resultados de la aplicación del algoritmo CONCOR a la matriz dicotomizada

\begin{tabular}{|c|l|}
\hline Grupos & \multicolumn{1}{|c|}{ Empresas } \\
\hline 1 & Abertis, Enagas, Acerinox, Fomento de Construcciones, NH Hoteles, Gas Natural, Repsol, La Caixa \\
2 & Telefónica, Endesa, ACS, Metrovacesa \\
3 & Banco Bilbao Vizcaya, Red Eléctrica, Altadis, Telefónica móviles, Iberdrola, Gamesa \\
4 & Cajamadrid, Iberia, Zeltia \\
5 & Grupo Ferrovial, Acciona, Banco Santander Central Hispano \\
6 & Mapfre, Arcelor, Popular, Sabadell, Amadeus \\
7 & Indra, TPI, Bankinter, Banesto, Inditex \\
8 & Sogecable, Unión Fenosa, Prisa \\
\hline
\end{tabular}

\subsection{Determinación de agrupaciones mediante CONCOR}

Para estudiar la estructura acudimos al algoritmo de caracterización estructural CONCOR. El algoritmo CONCOR (CONcordancia de CORrelaciones) es un tipo de agrupación en cluster que fue descrito por primera vez por McQuitty y Clark (1968) y redescubierto de forma independiente por Schwartz y Breiger (Schwartz, 1977). A partir de la matriz rectangular, el algoritmo reúne en una misma agrupación, mediante un proceso iterativo, los actores de la red con mayor semejanza en sus relaciones con el resto de actores. Para interpretar correctamente las agrupaciones resultantes, se ha determinado la matriz de densidades cruzadas entre todos los grupos. La dicotomización de la matriz reduce la red a una imagen simplificada de la intensidad de las relaciones intra y extra grupales.

Al aplicar CONCOR, se ha decidido limitar el número de iteraciones a tres, pues así se obtienen ocho agrupaciones, lo que permite una buena interpretación de los resultados. Los resultados de la aplicación del algoritmo a la matriz rectangular mediante el programa UCINET se muestran en la Tabla 3.

En la Figura I se muestra el grafo asociado a la matriz de densidad de cruzamientos entre grupos. Para una mejor interpretación del grafo, sólo se muestran los arcos con valores asociados por encima de la media.

\section{Discusión}

\section{I. Centralidad}

El examen de la Tabla I nos permite establecer diversas características de las empresas más centrales la red: las posiciones de centralidad para el gráfico ponderado y no ponderado las ocupan prácticamente las mismas empresas, excepción hecha de Prisa y Sogecable así como de Telefónica Móviles, que son más centrales en el grafo ponderado debido al peso de los arcos que los unen entre ellas y con Telefónica respectivamente. La presencia en la muestra de empresas pertenecientes a un mismo grupo (como es el caso de Prisa y Sogecable por un lado, y Telefónica y Telefónica Móviles por otro) hace que sea más significativo el indicador de centralidad de grado para el grafo dicotomizado. En el otro extremo de la lista hallamos los actores que se encuentran aislados del único componente que forma la red. Éstos son Amadeus Global (reservas para el sector turístico), Arcelor (Siderurgia), Mapfre (Seguros), Banco Popular y Banco de Sabadell (Entidades financieras). En cuanto a Amadeus y Arcelor, hay que indicar que éstas son las únicas empresas de la muestra con la sede social fuera de España.

Las empresas con mayor centralidad según la métrica de centralidad de proximidad son casi las mismas que las obtenidas con la centralidad de grado. En la Tabla

Figura |

Densidad de relaciones entre los grupos resultantes de la agrupación CONCOR (los nodos representan las filas de la Tabla 3)

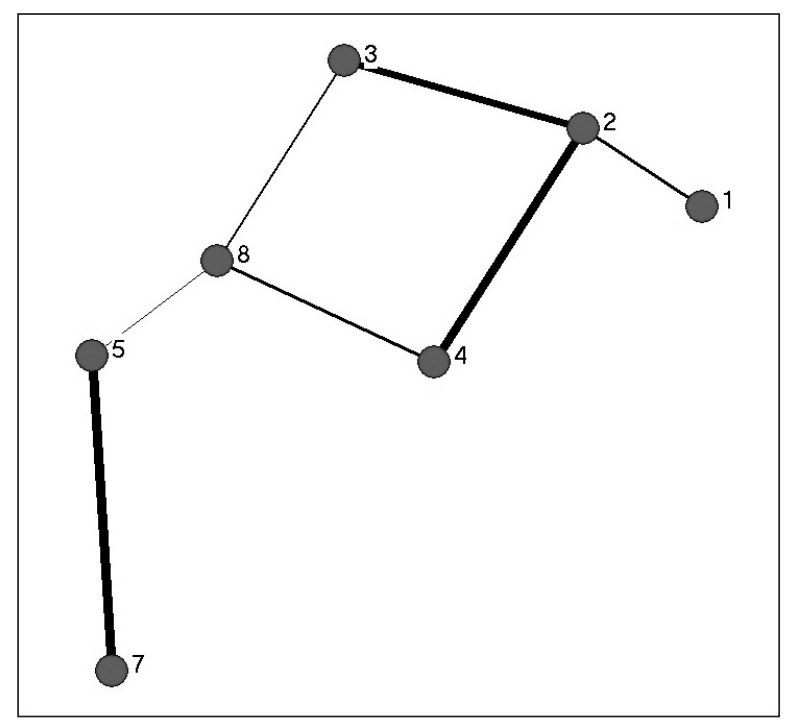


2, donde se indican las empresas más centrales según la métrica de proximidad, aparecen con mayor centralidad Telefónica Móviles y NH Hoteles (esta última no aparecía en la Tabla I), y tienen menor centralidad Endesa, Sogecable y Santander Central Hispano.

Los resultados obtenidos para la centralidad no muestran en ningún caso la existencia de bancos entre las posiciones más centrales de la red. Entran, pues, en conflicto con los resultados predichos por la teoría del control bancario, y presentan diferencias sustanciales con los del estudio de Aguilera (1998) con datos de 1993, en el que los bancos tenían un papel central en la red de consejerías cruzadas. No podemos descartar, sin embargo, que las entidades financieras (incluyendo las cajas de ahorros) ejerzan de alguna manera acciones de control sobre las empresas de las que son acreedoras (Byrd y Mizruchi, 2005) pero en esta red tal relación no se muestra en el intercambio de consejeros. También es posible que los bancos ejerzan otros modos de centralidad como la de intermediación (Freeman, 1979).

Buscando una característica común a los actores más centrales, resulta que los cuatro en encabezan las listas de centralidad basadas en los tres criterios analizados son empresas creadas por iniciativa estatal durante la dictadura (o se derivan directamente de éstas), y han sido posteriormente privatizadas. En el caso de Telefónica y de Iberia, ambas fueron creadas en los años 60, y los procesos de privatización finalizaron en el año 1997. Repsol y Enagas se crearon durante la reforma del sector energético español. La primera se crea en el Instituto Nacional de Hidrocarburos y se acaba de privatizar también en el año 1997, la segunda perteneció al INH y se acabó de privatizar en 1998.

En este punto debemos estar de acuerdo con Aguilera (1998) cuando propone que las consejerías cruzadas se deben observar desde una perspectiva histórica. En este caso, la permanencia de un núcleo de empresas privatizadas que mantienen fuertes lazos entre ellas y con el resto de las empresas puede ser el resultado de la forma en que se realizaron los procesos de privatización: mediante ofertas públicas de venta de acciones, adquiridas en su mayoría por otras entidades de la red.

Es también significativo que el sector predominante sea el energético, con Repsol, Enagas, Unión Fenosa y Endesa entre las empresas más centrales. De nuevo podemos buscar explicaciones en sucesos recientes: la reorganización del sistema energético español, especialmente el proceso de liberalización, llevan a que las posiciones de control de las empre- sas energéticas continúen en evolución, como lo demuestra la reciente lucha por el control de Endesa entre Gas Natural (otro de los actores de la red) y las extranjeras E.On y Enel.

\subsection{Agrupaciones de empresas mediante CONCOR}

La simplificación de la red ofrecida por el algoritmo CONCOR muestra un grupo I cuya principal característica es la densidad de intrarelaciones. El grupo incluye el único cluster de cinco actores totalmente conexo (es decir, en el que todos los consejos comparten consejeros con los demás): Abertis, Enagas, Gas Natural, Repsol y La Caixa. En cuanto a los otros tres miembros de la agrupación, NH Hoteles presenta múltiples relaciones con las cinco empresas del cluster, y Fomento de Construcciones y Acerinox están relacionados con Repsol. Ante la heterogeneidad del grupo (están presentes los sectores energético, construcción, hostelería...) cabe destacar que la única entidad financiera es La Caixa que tiene su sede central en Barcelona igual que Gas Natural y Abertis, también presentes en el grupo.

Si observamos la posición de las empresas privatizadas y de las entidades financieras de forma comparada según esta estructura, vemos que tanto unas como otras se encuentran repartidas en diferentes lugares, a excepción de los grupos Bankinter-Banesto, RepsolEnagas y los grupos 8 y 2 que no contienen ninguna entidad financiera. El resto de grupos (excepto el grupo 6, que reúne a los actores aislados) contienen una única entidad financiera cada uno. Esta peculiar parcelación apunta hacia la existencia de agrupaciones de la red en grupos heterogéneos con la presencia de diversos sectores donde los bancos no juegan un papel central. Estos clusters parecen vertebrarse alrededor de las empresas privatizadas que son las que tienen índices de centralidad local más elevados.

\section{Conclusiones}

Los resultados obtenidos del análisis de la red de consejerías a finales del año 2005 dan como resultado una organización peculiar de las empresas españolas cotizadas, que puede entenderse mejor interpretando estos resultados como una evolución de la situación descrita por Aguilera (1998). El primer hecho destacable es que las relaciones entre empresas de diferentes sectores son más intensas que entre empresas de un mismo sector. Ello se debe sin duda a la actuación de los organismos reguladores, los cuales evitan que las compras de participaciones 
entre empresas no reduzcan la competencia. Por tanto, es descartable que las consejerías cruzadas tengan como motivación la creación de monopolios. Salvo esta peculiaridad, el modelo de organización económica español tiene las características propias de un modelo continental, en el que no son los bancos, sino las empresas privatizadas, quienes tienen la mayor centralidad en la red. Comparando estos resultados con los de Aguilera, cabe concluir que los bancos españoles abandonan progresivamente el modelo de banco universal, para centrarse en la actividad comercial. Pero no hay que olvidar que los resultados de este estudio son una fotografía de un modelo en evolución. En el futuro, cabe esperar una centralidad aún menor de las entidades bancarias (La Caixa, única entidad bancaria con un grupo industrial visible en la red, ha manifestado su intención de vender sus participaciones industriales en breve), y una evolución aún incierta, pero dinámica, del sector energético. De la evolución futura de las empresas privatizadas depende que España adopte finalmente un modelo anglosajón de organización económica, o persistan trazas del actual modelo continental.

\section{Referencias}

AGUILERA, R. V. (1998). Directorship Interlocks in a Comparative Perspective: the Case of Spain, European Sociological Review, Vol. I 4, No. 4, pp. 319-342.

ASHAR, H., y SHAPIRO, J.Z. ( 1988). Measuring Centrality - A Note on Hackman Resource-Allocation Theory, Administrative Science Quarterly, Vol. 33, No. 2, pp. 275-283.

AZOFRA, V., y SANTAMARIA, M. (2004). El gobierno de las cajas de ahorro españolas. Universia Business Review, No. 2, pp. 48-59.

BORGATTI S. P.; EVERETT, M. G., y FREEMAN, L.C. (2002). Ucinet for Windows: Software for Social Network Analysis.

BYRD, D.T., y MIZRUCHI, M. S. (2005). Bankers on the Board and the Debt Ratio of Firms, Journal of corporate finance, Vol. II, Nos. I-2, pp. I29- 173.

DAVIS, G. F. ( 991 ). The Agents without Principles The Spread of the Poison Pill through the Intercorporate Network, Administrative Science Quarterly, Vol. 36, No. 4, pp. 583-6I3.

DIMAGGIO, P. J., y POWELL,W. W. (1983). The Iron Cage Revisited: Institutional Isomorphism and collective rationality in organizational fields, American Sociological Review, Vol. 48, pp. I47- 160.

FREEMAN, L. C. (1979). Centrality in Social Networks: Conceptual Clarification, Social Networks, Vol. I, No. 3, pp. 2I 5-239

FREEMAN, L. C.; BORGATTI, S. P., y WHITE, D. R. ( 199 |). Centrality in Valued Graphs: A Measure of
Betweenness Based on Network Flow, Social Networks, Vol. 13, No. I, pp. I4I-I54.

HAUNSCHILD, P. R. ( 1993). Interorganizational Imitation - The Impact of Interlocks on Corporate Acquisition Activity, Administrative Science Quarterly, Vol. 38, No. 4, pp. 564-592.

HAUNSCHILD, P. R., y BECKMAN, C. M. (1998). When do Interlocks Matter?:Alternate Sources of Information and Interlock Influence, Administrative Science Quarterly, Vol. 43, No. 4, pp. 8I 5-844.

MARSDEN, P. V. (2002). Egocentric and Sociocentric Measures of Network Centrality, Social Networks, Vol. 24, No. 4, pp. 407-422.

MCQUITTY, LL., y CLARK, J. A. (1968). Clusters from iterative intercolumnar correlation analysis, Educational and psychological measurement, Vol. 28, No. 2, pp. 21 I y ss.

MINTZ, B., y SCHWARTZ, M. ( 198 I). Interlocking directorates and interest group formation, American Sociological Review,Vol. 46, No. 6, pp. 85 I-869.

MIZRUCHI, M.S. ( 1996). What Do Interlocks Do? An Analysis, Critique, and Assessment of Research on Interlocking Directorates, Annual Review of Sociology, Vol. 22, pp. 27I-298.

O'HAGAN, S. B., y GREEN, M. B. (2004). Corporate Knowledge Transfer via Interlocking Directorates: a Network Analysis Approach, Geoforum, Vol. 35, pp. I27- I 39.

PENNINGS, J. M. ( 1980). Interlocking Directorates: Origins and Consequences of Connections Among Organizations' Boards of Directors, John Wiley and Sons, Ltd.

PROWSE, S. ( 1 994). Corporate governance in an international perspective: A survey of corporate control mechanisms among large firms in the united states, the United Kingdom, Japan and Germany. Economic Papers, Vol. 4I. Bank of International Settlements.

SCHWARTZ, J. E. (1977). An Examination of CONCOR and Related Methods for Blocking Sociometric Data, Sociological Methodology, Vol. 8, pp. 255-282.

SHLEIFER, A. Y., y VISHNY, R.W. (1997). A Survey of Corporate Governance, Journal of Finance, Vol. 52, No. 2, pp. 737-783.

SCOTT, J. (2000). Social Network Analysis: A Handbook, London: SAGE Publications.

USEEM, M. ( 1984). The Inner Circle, Oxford University Press.

ZAHRA, S. A., y PEARCE II, J. A. ( 1989). Boards of directors and corporate financial performance: A review and integrative model, Journal of Management, vol. 15, No. 2, pp. 29|-334.

ZAJAC, E. J. (1988). Interlocking Directorates as an Interorganizational Strategy - A Test of Critical Assumptions, Academy of Management Journal, Vol. 3 I , No. 2, pp. 428-438. 